\title{
High resolution spectroscopic analysis of seven giants in the bulge globular cluster NGC 6723
}

\author{
A. Rojas-Arriagada ${ }^{1}$, M. Zoccali ${ }^{2,3}$, S. Vásquez ${ }^{2,3,4}$, V. Ripepi ${ }^{5}$, I. Musella ${ }^{5}$, M. Marconi ${ }^{5}$, A. Grado ${ }^{5}$, and L. Limatola ${ }^{5}$ \\ ${ }^{1}$ Laboratoire Lagrange (UMR 7293), Université Nice Sophia Antipolis, CNRS, Observatoire de la Côte d'Azur, CS 34229 , \\ 06304 Nice Cedex 04, France \\ e-mail: arojas@oca.eu \\ ${ }^{2}$ Instituto de Astrofísica, Facultad de Física, Pontificia Universidad Católica de Chile, Av. Vicuña Mackenna 4860, Santiago, Chile \\ 3 Millennium Institute of Astrophysics, Av. Vicuña Mackenna 4860, 782-0436 Macul, Santiago, Chile \\ ${ }^{4}$ Museo Interactivo Mirador, Dirección de Educación, Av. Punta Arenas, 6711 La Granja, Santiago, Chile \\ 5 INAF-Osservatorio Astronomico di Capodimonte, via Moiariello 16, 80131 Naples, Italy
}

Received 12 September 2015 / Accepted 17 December 2015

\begin{abstract}
Context. Globular clusters associated with the Galactic bulge are important tracers of stellar populations in the inner Galaxy. High resolution analysis of stars in these clusters allows us to characterize them in terms of kinematics, metallicity, and individual abundances, and to compare these fingerprints with those characterizing field populations.

Aims. We present iron and element ratios for seven red giant stars in the globular cluster NGC 6723, based on high resolution spectroscopy.

Methods. High resolution spectra $(R \sim 48000)$ of seven K giants belonging to NGC 6723 were obtained with the FEROS spectrograph at the MPG/ESO $2.2 \mathrm{~m}$ telescope. Photospheric parameters were derived from $\sim 130 \mathrm{Fe}$ I and Fe II transitions. Abundance ratios were obtained from line-to-line spectrum synthesis calculations on clean selected features.

Results. An intermediate metallicity of $[\mathrm{Fe} / \mathrm{H}]=-0.98 \pm 0.08 \mathrm{dex}$ and a heliocentric radial velocity of $v_{\text {hel }}=-96.6 \pm 1.3 \mathrm{~km} \mathrm{~s} \mathrm{mere}^{-1}$ found for NGC 6723. Alpha-element abundances present enhancements of $[\mathrm{O} / \mathrm{Fe}]=0.29 \pm 0.18 \mathrm{dex},[\mathrm{Mg} / \mathrm{Fe}]=0.23 \pm 0.10 \mathrm{dex}$, $[\mathrm{Si} / \mathrm{Fe}]=0.36 \pm 0.05 \mathrm{dex}$, and $[\mathrm{Ca} / \mathrm{Fe}]=0.30 \pm 0.07$ dex. Similar overabundance is found for the iron-peak Ti with $[\mathrm{Ti} / \mathrm{Fe}]=$ $0.24 \pm 0.09$ dex. Odd-Z elements $\mathrm{Na}$ and $\mathrm{Al}$ present abundances of $[\mathrm{Na} / \mathrm{Fe}]=0.00 \pm 0.21 \mathrm{dex}$ and $[\mathrm{Al} / \mathrm{Fe}]=0.31 \pm 0.21 \mathrm{dex}, \mathrm{respec}-$ tively. Finally, the s-element $\mathrm{Ba}$ is also enhanced by $[\mathrm{Ba} / \mathrm{Fe}]=0.22 \pm 0.21$ dex.

Conclusions. The enhancement levels of NGC 6723 are comparable to those of other metal-intermediate bulge globular clusters. In turn, these enhancement levels are compatible with the abundance profiles displayed by bulge field stars at that metallicity. This hints at a possible similar chemical evolution with globular clusters and the metal-poor of the bulge going through an early prompt chemical enrichment.
\end{abstract}

Key words. stars: abundances - Galaxy: bulge - globular clusters: general - globular clusters: individual: NGC 6723

\section{Introduction}

The structure, age, and chemodynamical evolution of the Galactic bulge, together with its relation with other Galactic components, is currently debated. Several studies (Zoccali et al. 2008, 2014; Babusiaux et al. 2010; Hill et al. 2011; Ness et al. 2013; Rojas-Arriagada et al. 2014), based on a few hundred to several thousand stars, have constructed a new picture of the bulge as an extremely complex structure, including at least two stellar populations with different origins. In fact, bulge data shows chemodynamical distribution that are consistent with stars belonging to i) a narrow, metal-rich, boxy/peanut X-shaped component with bar-like kinematics, and ii) a broad metal-poor, kinematically hot population, which can be interpreted as a classical bulge coexisting with the Galactic bar.

The study of bulge field stars is complicated by the large spread in age and distance, and by the contamination from disk field stars overlapping in the lines of sight. In addition, the transition between internal (thick) disk and bulge is still not totally understood. In this context, the study of globular clusters as tracers of the bulge populations is a valuable tool because they provide clean points in the age-metallicity-distance distributions. Even if globular clusters are not necessarily representative of the field stellar populations, they must be included in the bulge formation scenario.

The globular cluster NGC 6723 is located at (J2000) $\alpha=$ $18^{\mathrm{h}} 59^{\mathrm{m}} 33.2^{\mathrm{s}}, \delta=-36^{\circ} 37^{\prime} 54^{\prime \prime}$, projected at about $-17^{\circ}$ from the Galactic center on the minor axis $\left(l=0.07^{\circ}, b=-17.299^{\circ}\right)$. According to the 2010 edition of the Harris (1996) catalog, NGC 6723 is rather bright $\left(M_{V}=-7.83 \mathrm{mag}\right)$, fairly large in size $\left(r_{\mathrm{t}}=10.5^{\prime}\right)$, and rather close to the Galactic center $\left(R_{\mathrm{GC}}=2.6 \mathrm{kpc}\right)$. Its proximity to the Galactic center makes it fall in the common definition of bulge cluster $\left(R_{\mathrm{GC}}<5 \mathrm{kpc}\right)$, although its nearly polar orbit suggests membership in the inner halo (Dinescu et al. 2003).

The most recent CMD of NGC 6723 available in the literature is by Lee et al. (2014). An updated census of the variable star content of NGC 6723 is performed from their high-precision $B V$ CCD photometry. Previous work in this direction goes back to the photographic work by Menzies (1974). From a Fourier 
decomposition analysis of the RR Lyrae variables, Lee et al. (2014) obtain $[\mathrm{Fe} / \mathrm{H}]=-1.23 \pm 0.11$ dex and $E(B-V)=$ $0.063 \pm 0.015 \mathrm{mag}$ with a distance modulus of $(m-M)_{0}=$ $14.65 \pm 0.05 \mathrm{mag}$.

On the other hand, spectroscopic measurements characterize NGC 6723 as an intermediate metallicity cluster. A high metallicity of $[\mathrm{Fe} / \mathrm{H}]=-0.7$ dex was obtained by Smith (1981) from low resolution $(R=1300)$ spectra of five RR Lyrae stars using the $\delta S$ parameter. Rutledge et al. (1997) obtained $[\mathrm{Fe} / \mathrm{H}]=$ -1.09 dex from calcium triplet measurements, while Kraft \& Ivans (2003) derived a metallicity of $[\mathrm{Fe} / \mathrm{H}]=-1.12$ dex by recalibrating the Rutledge et al. (1997) value. Additionally, two studies perform a chemical characterization of NGC 6723, based on samples of high resolution spectra. The results of Fullton \& Carney (1996) come from the analysis of a small sample of three RGB stars at $R \sim 33000$, deriving mean values of $[\mathrm{Fe} / \mathrm{H}]=-1.26 \mathrm{dex},[\mathrm{Ca} / \mathrm{Fe}]=0.33 \mathrm{dex},[\mathrm{Ti} / \mathrm{Fe}]=0.24 \mathrm{dex}$, and $[\mathrm{Si} / \mathrm{Fe}]=0.68 \mathrm{dex}$. Unfortunately, this work has never been published in a refereed journal, so details of the analysis are not publicly available. The recent work of Gratton et al. (2015) focus on the chemical analysis of a statistically significant sample of 30 red horizontal branch (RHB) and 17 blue horizontal branch (BHB) stars, observed at a maximum resolution of $R \sim 18700$. From this sample, they inferred an average radial velocity of $V_{\text {rad }}=-95.8 \pm 0.6 \mathrm{~km} \mathrm{~s}^{-1}$ and mean values of $[\mathrm{Fe} / \mathrm{H}]=-1.22 \pm 0.08 \operatorname{dex},[\mathrm{O} / \mathrm{Fe}]=0.53 \pm 0.09,[\mathrm{Na} / \mathrm{Fe}]=$ $0.13 \pm 0.09,[\mathrm{Mg} / \mathrm{Fe}]=0.51 \pm 0.06,[\mathrm{Si} / \mathrm{Fe}]=0.60 \pm 0.08$ $[\mathrm{Ca} / \mathrm{Fe}]=0.81 \pm 0.13$, and $[\mathrm{Ba} / \mathrm{Fe}]=0.75 \pm 0.17$ dex for NGC 6723. Complementary high resolution spectroscopic studies, targeting globular cluster samples at different evolutionary stages, are important to accurately characterize their chemical signatures and shed light on their eventual complexities. This is especially important in that our current understanding of globular clusters departs from the classical hypothesis of simple stellar populations.

We carried out an observing campaign at the $2.2 \mathrm{~m}$ telescope at ESO La Silla Observatory, to obtain time series photometry with the Wide Field Imager (WFI; Baade et al. 1999) and high resolution spectroscopy with the Fiber-fed Extended Range Optical Spectrograph (FEROS; Kaufer et al. 1999). We analyze spectra of eight giants (from which seven turn out to be cluster candidates), deriving iron and element ratios. The analysis of the CMD and variable star content of the cluster will be published in a forthcoming paper.

The paper is organized as follows: the observations are described in the next section, while initial photometric stellar parameters are presented in Sect. 3. The adopted line list is described in Sect. 4. Iron abundances are derived in Sect. 5. Section 6 discusses distance and a foreground interstellar reddening values derived from the spectroscopic parameters. In Sect. 7 we present our derived abundance ratios. Discussion and conclusions are in Sect. 8.

\section{The data}

Spectra for eight stars in the globular cluster NGC 6723 were obtained with the FEROS spectrograph (Kaufer et al. 1999) at the MPG/ESO $2.2 \mathrm{~m}$ telescope (La Silla Observatory), as part of the ESO program 085.D-0143. FEROS spectra are in the range 3500-9200 $\AA$, spread over 39 echelle orders with a resolution of $R \sim 48000$. Two fibers allowed us to obtain a stellar spectrum plus a sky simultaneously during a single exposure. The observed sample stars, at the sky positions depicted in Fig. 1, were

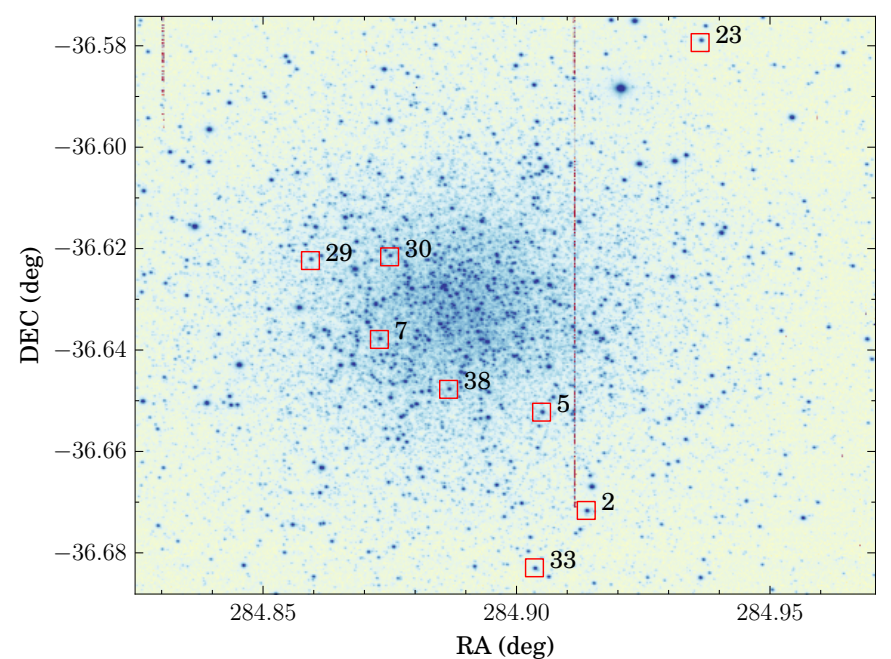

Fig. 1. Finding chart showing the location of the 8 stars observed in NGC 6723 . The background image correspond to a $\sim 8.76^{\prime} \times 6.84^{\prime}$ section of a WFI image in the BB\#V/89-ESO843 filter.
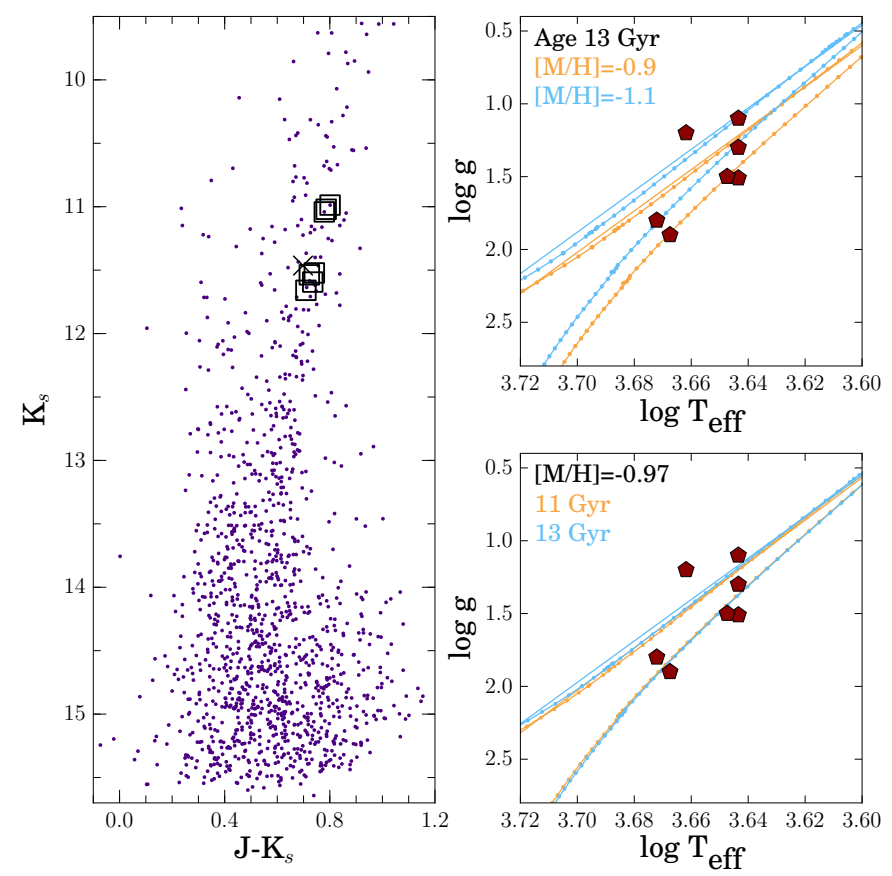

Fig. 2. Left panel: $K_{\mathrm{s}}$ vs. $J-K_{\mathrm{s}} 2 \mathrm{MASS}$ based CMD of the cluster. The spectroscopic targets are marked with black empty squares. The field contaminant star is marked with a cross symbol. Right panels: $\log (g)$ vs. $T_{\text {eff }}$ diagrams for the spectroscopic sample. PARSEC (Bressan et al. 2012) 13 Gyr isochrones for metallicity $[\mathrm{M} / \mathrm{H}]=-0.9$ dex and $[\mathrm{M} / \mathrm{H}]=$ -1.1 dex are overplotted in the upper panel, while two isochrones of 11 and $13 \mathrm{Gyr}$ with the cluster metallicity in the lower panel.

selected from the $\mathrm{K}$ giant region of the cluster $\mathrm{CMD}$, as shown in the left panel of Fig. 2. Table 1 lists the coordinates, 2MASS IDs (Skrutskie et al. 2006) and the $V J H K_{\text {s }}$ photometry of the observed stars. $J, H$, and $K_{\mathrm{s}}$ magnitudes come from $2 \mathrm{MASS}$, while $V$ corresponds to a visual magnitude from the UCAC3 catalog (Zacharias et al. 2010).

The spectra were flat-fielded, optimally extracted, wavelength calibrated, and corrected to heliocentric system of reference (barycentric correction) with the standard FEROS-DRS 
Table 1. Identifications, positions, and magnitudes of the target stars.

\begin{tabular}{lccccccc}
\hline \hline Star ID & 2MASS ID & $\alpha(\mathrm{J} 2000.0)$ & $\delta(\mathrm{J} 2000.0)$ & $J$ & $H$ & $K_{\mathrm{s}}$ & $V_{\mathrm{UCAC} 3}$ \\
\hline$\# 1$ & $18593930-3640179$ & 284.913763 & -36.67165 & 11.788 & 11.124 & 10.987 & 14.079 \\
$\# 2$ & $18593718-3639082$ & 284.904941 & -36.652302 & 11.806 & 11.163 & 11.020 & 14.048 \\
$\# 3$ & $18592950-3638165$ & 284.872956 & -36.637917 & 11.820 & 11.089 & 11.041 & 14.051 \\
$\# 4$ & $18592624-3637204$ & 284.859371 & -36.622349 & 12.263 & 11.611 & 11.522 & 14.375 \\
$\# 5$ & $18592998-3637177$ & 284.874931 & -36.621597 & 12.259 & 11.611 & 11.537 & 14.385 \\
$\# 6$ & $18593684-3640585$ & 284.903519 & -36.682938 & 12.329 & 11.696 & 11.594 & 14.407 \\
$\# 7$ & $18593278-3638519$ & 284.886611 & -36.647755 & 12.368 & 11.737 & 11.659 & 14.515 \\
$\# 8$ & $18594469-3634457$ & 284.936217 & -36.579371 & 12.162 & 11.397 & 11.464 & 14.524 \\
\hline
\end{tabular}

Table 2. Log of spectroscopic observations.

\begin{tabular}{lcccccccc}
\hline \hline Target & Date & UTC & $\begin{array}{c}\text { Exp } \\
(\mathrm{s})\end{array}$ & Airmass & $\begin{array}{c}\text { Seeing } \\
\left({ }^{\prime \prime}\right)\end{array}$ & $(S / N) / \mathrm{px}$ & $\begin{array}{c}v_{\text {hel }} \\
\mathrm{km} \mathrm{s}^{-1}\end{array}$ & $\begin{array}{c}\text { Corr }_{\text {hel }} \\
\mathrm{km} \mathrm{s}^{-1}\end{array}$ \\
\hline$\# 1$ & $2010-05-05$ & $09: 49: 21.971$ & 2700 & 1.057 & 0.93 & 61 & -96.65 & 24.31 \\
& $2010-05-06$ & $08: 10: 16.920$ & 5400 & 1.014 & 1.00 & & -96.52 & 24.20 \\
$\# 2$ & $2010-05-05$ & $05: 46: 51.973$ & 5400 & 1.160 & 1.22 & 54 & -90.01 & 24.67 \\
$\# 3$ & $2010-05-05$ & $07: 30: 43.969$ & 5400 & 1.021 & 1.11 & 58 & -100.38 & 24.52 \\
$\# 4$ & $2010-06-22$ & $01: 24: 06.707$ & 4600 & 1.450 & 1.47 & 37 & -99.78 & 6.14 \\
& $2010-06-22$ & $03: 11: 28.710$ & 4600 & 1.103 & 1.77 & & -99.82 & 6.01 \\
$\# 5$ & $2010-06-22$ & $04: 33: 50.710$ & 4500 & 1.018 & 1.56 & 42 & -96.16 & 5.89 \\
& $2010-06-22$ & $05: 50: 02.710$ & 4500 & 1.027 & 1.54 & & -96.20 & 5.75 \\
$\# 6$ & $2010-06-22$ & $07: 42: 12.708$ & 4500 & 1.211 & 1.47 & 26 & -94.08 & 5.57 \\
& $2010-06-22$ & $08: 58: 04.707$ & 4500 & 1.540 & 1.62 & & -94.25 & 5.46 \\
& $2010-06-23$ & $04: 18: 17.468$ & 4500 & 1.024 & 0.84 & 42 & -98.84 & 5.44 \\
& $2010-06-25$ & $05: 06: 03.250$ & 3600 & 1.011 & 1.39 & & -99.01 & 5.31 \\
$\# 8$ & $2010-06-25$ & $06: 06: 55.251$ & 3600 & 1.043 & 1.55 & & -99.05 & 4.29 \\
& $2010-06-23$ & $01: 12: 15.468$ & 4500 & 1.500 & 1.07 & 31 & 7.29 & 5.71 \\
& $2010-06-23$ & $02: 28: 33.467$ & 4500 & 1.191 & 0.91 & & 7.35 & 5.63 \\
\hline
\end{tabular}

Notes. For stars with several individual exposures, the signal-to-noise value corresponds to that of the final averaged spectrum.

pipeline ${ }^{1}$ based on MIDAS. For most of the stars, the total exposure time was split into two or more exposures to remove cosmic rays. A log of observations is listed in Table 2. We use the IRAF ${ }^{2}$ task skytweak to remove the sky emission lines from the individual spectra with the corresponding companion sky fiber. After applying the continuum task and a cubic spline fit, we normalized the stellar continuum to the unit.

The radial velocity of each individual spectra was derived by cross-correlation (IRAF task fxcor) with a synthetic template. This template was generated adopting stellar parameters typical of $\mathrm{K}$ giant stars, i.e. $T_{\text {eff }}=4500 \mathrm{~K},[\mathrm{Fe} / \mathrm{H}]=-1.0$ dex, $\log (g)=1.8 \mathrm{dex}$, and $v_{\mathrm{t}}=1.4 \mathrm{~km} \mathrm{~s}^{-1}$. The corrected final restframe spectra were obtained by applying the velocity correction with dopcor. Post-processed spectra corresponding to individual exposures of each star were then median combined. A mean heliocentric radial velocity of $v_{\text {hel }}=-96.6 \pm 1.3 \mathrm{~km} \mathrm{~s}^{-1}$ was found for NGC 6723, which is in excellent agreement with the value of $v_{\text {hel }}=-94.5 \pm 3.6 \mathrm{~km} \mathrm{~s}^{-1}$ reported in the compilation by Harris (1996). One of the program stars (\#8) is not a member of the cluster according to its radial velocity. The contaminant nature of this star is confirmed by the metallicity determinations presented in Sect. 5. The propagation of internal errors (the velocity errors from the cross-correlation) of individual stars gives $\sigma_{\text {internal }}=0.62$. The r.m.s velocity scatter from cluster members

\footnotetext{
1 http://www.eso.org/sci/facilities/lasilla/ instruments/feros/tools/DRS.html

2 IRAF is distributed by the National Optical Astronomy Observatory, which is operated by the Association of Universities for Research in Astronomy (AURA) under cooperative agreement with the National Science Foundation.
}

correspond to $3.37 \mathrm{~km} \mathrm{~s}^{-1}$. These values imply an internal velocity dispersion of $3.3 \mathrm{~km} \mathrm{~s}^{-1}$ for NGC 6723 . In comparison, Gratton et al. (2015) quote a value of $4.3 \mathrm{~km} \mathrm{~s}^{-1}$.

Signal-to-noise $(\mathrm{S} / \mathrm{N})$ ratios were measured in the final coadded spectra at several wavelength regions spanning $2-3 \AA$. The values reported in Table 2, correspond to the mean of these measurements.

\section{Photometric stellar parameters}

We estimate photometric atmospheric parameters as a first guess to perform a more precise spectroscopic analysis. Effective temperatures $T_{\text {eff }}$ were derived from the $V-J, V-H, V-K$, and $J-K$ colors using the recent calibrations by González Hernández \& Bonifacio (2009). These calibrations were constructed by performing a fully self-consistent infrared flux method in the 2MASS photometric system, and thus are the best choice available in the literature. To use them, we adopt a metallicity of $[\mathrm{Fe} / \mathrm{H}]=-1.0$ dex and a mean reddening of $E(B-V)=$ 0.05 mag for NGC 6723, as taken from the 2010 edition of the Harris (1996) compilation. We adopted the extinction law given by Cardelli et al. (1989), i.e., $R_{V}=A_{V} / E(B-V)=3.1$, $E(V-J) / E(B-V)=2.26, E(V-H) / E(B-V)=2.51$, $E(V-K) / E(B-V)=2.75$, and $E(J-K) / E(B-V)=0.52$.

The derived photometric effective temperatures are listed on the left side of Table 3 .

Using those $T_{\text {eff }}$ values, we derive gravity from the classical relation

$\log g_{\star}=4.44+4 \log \frac{T_{\star}}{T_{\odot}}+0.4\left(M_{\mathrm{bol} \star}-M_{\mathrm{bol} \odot}\right)+\log \frac{M_{\star}}{M_{\odot}}$,

A95, page 3 of 10 
Table 3. Photometric temperatures derived from $J-K, V-J, V-H$ and $V-K$ colors, bolometric corrections, corresponding derived gravities and final spectroscopic parameters of the target stars.

\begin{tabular}{|c|c|c|c|c|c|c|c|c|c|c|c|c|}
\hline \multirow[b]{2}{*}{ Star } & \multicolumn{7}{|c|}{ Photometric parameters } & \multicolumn{5}{|c|}{ Spectroscopic parameters } \\
\hline & $\begin{array}{c}T(J-K)^{a} \\
\mathrm{~K}\end{array}$ & $\begin{array}{c}T(V-J)^{b} \\
\mathrm{~K}\end{array}$ & $\begin{array}{c}T(V-H)^{c} \\
\mathrm{~K}\end{array}$ & $\begin{array}{c}T(V-K)^{d} \\
\mathrm{~K}\end{array}$ & $\begin{array}{l}B C_{v} \\
\text { mag }\end{array}$ & $\begin{array}{c}\log (g) \\
\operatorname{dex}\end{array}$ & $\begin{array}{c}T_{\mathrm{eff}} \\
\mathrm{K}\end{array}$ & $\begin{array}{c}\log (g) \\
\operatorname{dex}\end{array}$ & $\begin{array}{c}{[\mathrm{Fe} \mathrm{I} / \mathrm{H}]} \\
\operatorname{dex}\end{array}$ & $\begin{array}{c}{[\mathrm{Fe} \mathrm{II} / \mathrm{H}]} \\
\operatorname{dex}\end{array}$ & $\begin{array}{c}v_{\mathrm{t}} \\
\mathrm{km} \mathrm{s}^{-1}\end{array}$ & $\begin{array}{c}V_{\text {hel }} \\
\mathrm{km} \mathrm{s}^{-1}\end{array}$ \\
\hline$\# 1$ & 4359 & 4353 & 4261 & 4268 & -0.29 & 1.48 & 4400 & 1.51 & -0.96 & -0.95 & 1.3 & -96.586 \\
\hline \#2 & 4399 & 4397 & 4309 & 4310 & -0.27 & 1.49 & 4400 & 1.30 & -1.05 & -1.04 & 1.3 & -90.010 \\
\hline \#3 & 4417 & 4407 & 4257 & 4322 & -0.27 & 1.49 & 4440 & 1.50 & -0.99 & -1.05 & 1.4 & -100.377 \\
\hline \#4 & 4522 & 4520 & 4395 & 4431 & -0.22 & 1.69 & 4700 & 1.80 & -0.93 & -0.93 & 1.4 & -99.787 \\
\hline \#5 & 4576 & 4506 & 4388 & 4436 & -0.22 & 1.70 & 4650 & 1.90 & -0.91 & -0.94 & 1.3 & -95.182 \\
\hline \#6 & 4539 & 4555 & 4435 & 4461 & -0.21 & 1.72 & 4400 & 0.60 & -0.87 & -0.84 & 0.9 & -94.164 \\
\hline \#7 & 4614 & 4486 & 4385 & 4430 & -0.22 & 1.75 & 4400 & 1.10 & -1.10 & -1.09 & 1.0 & -98.966 \\
\hline \#8 & 4646 & 4293 & 4150 & 4289 & -0.28 & 1.68 & 4500 & 1.90 & -0.29 & -0.31 & 1.0 & 7.322 \\
\hline
\end{tabular}

Notes. ${ }^{(a)}$ Calibration standard deviation: 94 K. ${ }^{(b)}$ Calibration standard deviation: 18 K. ${ }^{(c)}$ Calibration standard deviation: 23 K. ${ }^{(d)}$ Calibration standard deviation: $23 \mathrm{~K}$.

adopting $T_{\odot}=5770 \mathrm{~K}, M_{\star}=0.85 M_{\odot}, M_{\text {bol } \odot}=4.75$, a distance modulus of $(m-M)_{V}=14.84$ mag for NGC 6723 (Harris compilation), and the reddening already used to derive the temperatures. The bolometric corrections from Alonso et al. (1999) and the estimated gravities are given in the last two columns of the left side of Table 3.

\section{The line list}

The Fe I line list and respective oscillator strengths used here includes $\sim 130$ transitions, and are described in Zoccali et al. (2008). Also included in the list are ten Fe II transitions carefully selected to have a minimum impact from blends in $\mathrm{K}$ giants. In our analysis, we only used a part of the reddest portion of the FEROS spectrum from 5500 to $6800 \AA$. At shorter wavelengths, line density is high and some molecular bands are present, making the continuum determination difficult. Because of the small amount of available lines, the $\mathrm{Fe}$ II constraint to $[\mathrm{Fe} / \mathrm{H}]$ is sensitive to the error introduced by blended and small undefined lines. To ensure a clean analysis, we visually examined each spectrum to check the quality of the Fe II lines, eliminating those lines that are too small or suspicious blending cases from the subsequent analysis. In the analysis, we used only Fe I and Fe II lines with equivalent widths $E W<200 \mathrm{~m} \AA$.

Lines and respective atomic constants for odd- $\mathrm{Z}$ elements $\mathrm{Al}, \mathrm{Na}, \alpha$-elements $\mathrm{Mg}, \mathrm{Si}, \mathrm{Ca}, \mathrm{Ti}$, and heavy element $\mathrm{Ba}$ were adopted from Zoccali et al. (2008). The specific set of lines was verified to be clean from blends from telluric lines in our target stars. The damping constants $C_{6}$ were adopted from Coelho et al. (2005). They were computed where possible, and in particular for most of the Fe I lines, using the collisional broadening theory of Barklem et al. (1998, 2000). In order to check the internal consistency of our chemical analysis with this line list, we took a solar spectrum from the FEROS archive, reducing it in the same way as the target stars. Spectrum synthesis calculations were then used to reproduce the solar spectrum. In those cases where the fit to the Sun could be improved, the oscillator strength value $\log (g f)$ of the respective line was changed until a best fit was obtained. We adopted the oscillator strength $\log (g f)=-9.716$ derived by Allende Prieto et al. (2001) for the forbidden oxygen line at $6300 \AA$. Solar abundances were adopted from Grevesse \& Sauval (1998), except for oxygen, where a value of $\epsilon(O)=8.77$ was adopted, as recommended by Allende Prieto et al. (2001) for the use of 1D model atmospheres.

\section{Iron abundances}

Equivalent widths were measured in the coadded spectra via the automatic code DAOSPEC (Stetson \& Pancino 2008).

Model atmospheres were interpolated from the MARCS grid of spherical models ${ }^{3}$ described in Gustafsson et al. (2003). A LTE analysis was performed with an improved and updated version of the classical code presented in Spite (1967), using the atmospheric models and EW measured for the Fe I and Fe II lines in the list. A solar iron abundance of $\epsilon(\mathrm{Fe})=7.50$ (Grevesse $\&$ Sauval 1998) was adopted. The stellar parameters were derived through an iterative process, starting from the photometric temperature and gravity as an initial guess. Temperature was further constrained by imposing the excitation equilibrium of Fe I lines, namely, that lines of different excitation potential $\chi_{\text {ex }}$ gave the same iron abundance. The measured Fe II lines enabled us to derive gravity by imposing ionization equilibrium (Fe I and Fe II abundances in agreement). Microturbulence velocity $v_{\mathrm{t}}$ was determined by eliminating the trend of $\mathrm{Fe} \mathrm{I}$ abundances versus $\mathrm{EW}_{\mathrm{p}}$. We used predicted $\mathrm{EW}_{\mathrm{p}}$ instead of observed $\mathrm{EW}$ to avoid the introduction of systematics due to the correlation of errors in EW and metallicity, as explained in Zoccali et al. (2008). Iron abundances were obtained as the weighted mean of the line-by-line measurements, where the weight associated with each line is the inverse square of its abundance error, as derived from the error in the EW measurement. The procedure is illustrated for star \#5 in Fig. 3. Final spectroscopic parameters $T_{\text {eff }}$, $\log (g),[\mathrm{Fe} / \mathrm{H}]$, and $v_{\mathrm{t}}$ are given in the right side of Table 3 . The star final iron abundance $[\mathrm{Fe} / \mathrm{H}]$ is taken as the average between values estimated from Fe I and Fe II lines.

It is clear from Table 3 that star \#8, previously discarded as a member of the cluster because of its radial velocity, has also a metallicity significantly higher than the remaining sample stars. Having confirmed its contaminant nature, we exclude it from the following analysis.

An average metallicity of $[\mathrm{Fe} / \mathrm{H}]=-0.98 \pm 0.08$ dex is derived for NGC 6723.

http://www .marcs.astro.uu.se/ 

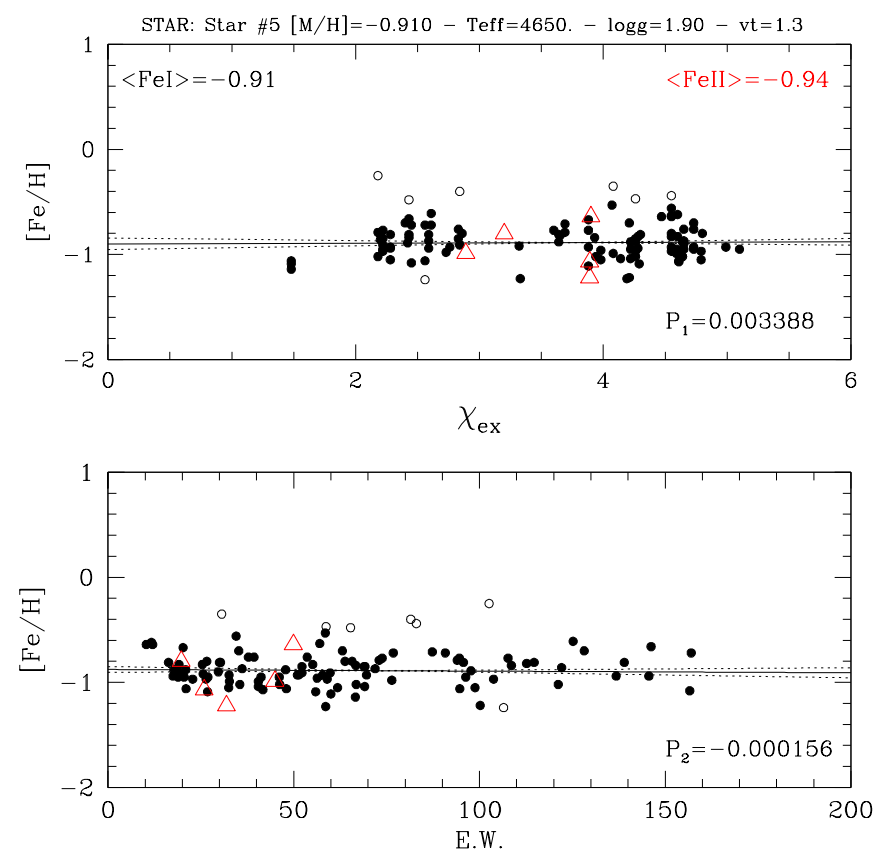

Fig. 3. Fe I (black solid dots) and Fe II (red open triangles) abundances versus $\chi_{\mathrm{ex}}(\mathrm{eV})$ and $\mathrm{EW}_{\mathrm{p}}(\mathrm{m} \AA)$ for star \#5. Open circles stand for $\mathrm{Fe}$ I lines excluded from computation of the mean abundance using a $\sigma$ clipping criterion. Temperature and microturbulence are estimated by eliminating the trend of $\mathrm{Fe} I$ abundance with respect to $\chi_{\mathrm{ex}}$ and $\mathrm{EW}_{\mathrm{p}}$.

Table 4. Fe I and Fe II uncertainties induced by a change of $\Delta T_{\text {eff }}=$ $100 \mathrm{~K}, \Delta \log (g)=0.2 \mathrm{dex}, \Delta v_{\mathrm{t}}=0.2 \mathrm{~km} \mathrm{~s}^{-1}$, and corresponding total error.

\begin{tabular}{llcccc}
\hline \hline \multirow{2}{*}{ Star } & Specie & $\Delta T$ & $\Delta \log (g)$ & $\Delta v_{\mathrm{t}}$ & $\left(\sum x^{2}\right)^{1 / 2}$ \\
\hline$\# 1$ & {$[\mathrm{Fe} / \mathrm{H}](\mathrm{I})$} & -0.05 & -0.03 & 0.09 & 0.11 \\
& {$[\mathrm{Fe} / \mathrm{H}](\mathrm{II})$} & 0.13 & -0.11 & 0.05 & 0.18 \\
$\# 2$ & {$[\mathrm{Fe} / \mathrm{H}](\mathrm{I})$} & 0.06 & 0.03 & -0.10 & 0.12 \\
& {$[\mathrm{Fe} / \mathrm{H}](\mathrm{II})$} & -0.13 & 0.10 & -0.06 & 0.18 \\
$\# 3$ & {$[\mathrm{Fe} / \mathrm{H}](\mathrm{I})$} & 0.04 & 0.02 & -0.09 & 0.10 \\
& {$[\mathrm{Fe} / \mathrm{H}](\mathrm{II})$} & -0.08 & -0.16 & 0.00 & 0.18 \\
$\# 4$ & {$[\mathrm{Fe} / \mathrm{H}](\mathrm{I})$} & 0.09 & 0.03 & -0.08 & 0.12 \\
& {$[\mathrm{Fe} / \mathrm{H}](\mathrm{II})$} & -0.07 & 0.10 & -0.05 & 0.13 \\
$\# 5$ & {$[\mathrm{Fe} / \mathrm{H}](\mathrm{I})$} & 0.09 & 0.02 & -0.08 & 0.12 \\
& {$[\mathrm{Fe} / \mathrm{H}](\mathrm{II})$} & -0.10 & 0.10 & -0.06 & 0.15 \\
$\# 6$ & {$[\mathrm{Fe} / \mathrm{H}](\mathrm{I})$} & 0.07 & 0.02 & -0.18 & 0.19 \\
& {$[\mathrm{Fe} / \mathrm{H}](\mathrm{II})$} & -0.12 & 0.10 & -0.10 & 0.19 \\
$\# 7$ & {$[\mathrm{Fe} / \mathrm{H}](\mathrm{I})$} & 0.11 & 0.10 & -0.06 & 0.16 \\
& {$[\mathrm{Fe} / \mathrm{H}](\mathrm{II})$} & 0.11 & 0.35 & 0.17 & 0.40 \\
$\# 8$ & {$[\mathrm{Fe} / \mathrm{H}](\mathrm{I})$} & 0.03 & 0.03 & -0.15 & 0.16 \\
& {$[\mathrm{Fe} / \mathrm{H}](\mathrm{II})$} & -0.18 & 0.12 & -0.09 & 0.23 \\
\hline
\end{tabular}

We estimate that an uncertainty of $\pm 100 \mathrm{~K}$ in $T_{\text {eff }}$ is well inside the error in the slope of the $[\mathrm{Fe} / \mathrm{H}]$ vs. $\chi_{\text {ex }}$ trend used to constrain the temperature (Fig. 3). Similarly, by imposing a flat trend of Fe I line abundances as a function of $\mathrm{EW}_{\mathrm{p}}$, we can constrain $v_{\mathrm{t}}$ with a precision of $\pm 0.2 \mathrm{~km} \mathrm{~s}^{-1}$. Ionization equilibrium allows us to constrain $\log (g)$ within \pm 0.2 dex. We estimate errors in metallicity from Fe I and Fe II lines allowing a variation of $\Delta T_{\text {eff }}=100 \mathrm{~K}, \Delta \log (g)=0.2 \mathrm{dex}$, and $\Delta v_{\mathrm{t}}=0.2 \mathrm{~km} \mathrm{~s}^{-1}$, and quantifying their impact in the final value. The induced error corresponds to the difference between an abundance derived with the altered and nominal models. Individual errors are listed in Cols. 3-5 of Table 4 and the total error is listed in Col. 6. Average errors associated with $\mathrm{Fe} I$ and $\mathrm{Fe}$ II lines are 0.14 and 0.21 dex, respectively. These error bars are larger than the statistical uncertainty of 0.08 in the average cluster metallicity derived from the star individual values. This means that our assumed uncertainties on $T_{\text {eff }}, \log (g)$ and $v_{\mathrm{t}}$ are, in fact, probably overestimated given the good data quality of our spectra. In view of that, we adopt the statistical uncertainty of $0.08 \mathrm{dex}$ as the error bar associated with the cluster metallicity estimate.

\section{Isochrone fitting: distance and reddening}

Since they are cluster members, our target stars are located essentially at the same distance and are expected to have similar reddening values aside from small differential extinction across the cluster area. We use our derived spectroscopic parameters and available photometry to compute distances for our sample via an isochrone fitting technique. The algorithm (Rojas-Arriagada et al., in prep.) makes use of the PARSEC set of isochrones to compute given atmospheric parameters and metallicity, a theoretical absolute magnitude $M_{\lambda}$ in a specific photometric band. Given $T_{\text {eff }}, \log (g)$, metallicity and corresponding errors, the algorithm selects a set of isochrones of all ages matching metallicity values inside the error bar. A Monte Carlo sampling is generated in the $T_{\text {eff- }} \log (g)$ plane by considering the ellipsoid given by the respective errors. The distribution is projected onto the isochrones via closest match with a distance normalized by the errors as a metric. From the best match of each Monte Carlo sample, we obtain a theoretical absolute magnitude in the photometric band of interest. From this set of values, weighted by matching distance and evolutionary speed $^{4}$, a probability distribution function $(p d f)$ for $M_{\lambda}$ is constructed. A final absolute magnitude value for the star under analysis can be obtained as the mode or median of such distribution. Additionally, if we have photometry in $J$ and $K$ bands, a reddening estimate can be computed simultaneously with the distance. From the computed absolute magnitude $M_{\lambda}$, a distance modulus $\mu$, and then a distance is calculated. The distance modulus is corrected by reddening either using an input value or the value computed in-situ. Tests have shown that no significant differences arise from this choice.

Using the procedure described above, we compute distances and foreground interstellar reddening for all the stars in the sample. The adopted errors in atmospheric parameters are the nominal parameters: $\Delta T_{\text {eff }}=100 \mathrm{~K}, \Delta \log (g)=0.2 \mathrm{dex}$, and $\Delta[\mathrm{Fe} / \mathrm{H}]=0.15 \mathrm{dex}$. We include the nonmember star \#8 as a consistency test; its resulting distance is the smallest of the sample, confirming its foreground contaminant nature. For each star, we have a set of four distance estimates because of the two 2MASS photometric bands employed ( $J$ and $K_{\mathrm{s}}$ ) and the two ways to include reddening (external or estimated in-situ). In each case, we average results coming from $J$ and $K_{\mathrm{s}}$ passbands as they have in fact a very small dispersion $(\sigma \sim 0.22 \mathrm{kpc}$ in both cases). The two sets of final individual star distances are depicted by blue circles (determined using external reddening figures) and red crosses (determined using reddening calculated in-situ) at the bottom of Fig. 4. Given the error figures estimated

\footnotetext{
4 This quantity is related to the mass differences $\Delta m$ between successive points in the isochrone, which gives in fact a proxy for the evolutionary speed of model stars at different evolutionary stages.
} 


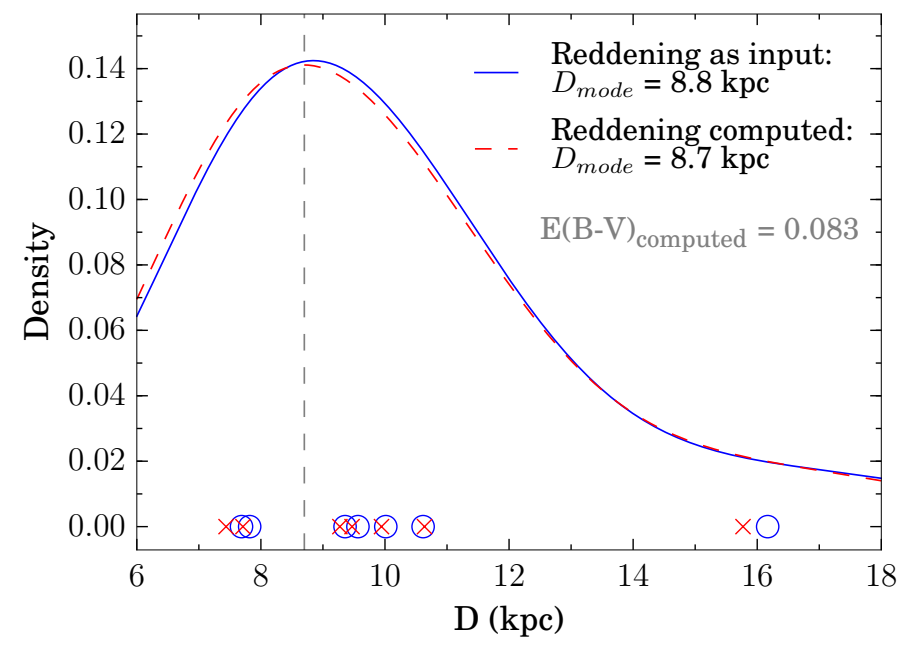

Fig. 4. Distance probability function of NGC 6723. Symbols at the bottom stand for the distances determined for individual stars. A blue solid line depicts the global $p d f$ generated from individual distances (depicted as blue circles) determined using reddening values from external measurements. On the other hand, a red dashed line depicts the global $p d f$ generated from individual distances (depicted by the red crosses) determined using reddening values computed simultaneously with the respective distances. In each case, errors on individual distance measurements are considered to compute the global cluster distance $p d f$, assuming each individual star as a Gaussian random variable around the measured value. The modes of the $p d f \mathrm{~s}$ are quoted, and provide our best estimates for the distance of NGC 6723. The estimated average reddening toward the cluster is also quoted in the panel. A vertical gray dashed line indicates the distance of NGC 6723 as given in the Harris (1996) compilation.

by the code for each distance, we can consider each star as a Gaussian random variable and then compute the global cluster distance $p d f$ as the superposition of individual contributions. The result of this is shown by the blue (external reddening) and red (reddening computed in-situ) curves. We can see that the selected method to include reddening in our calculations has, as anticipated, a low impact on the final cluster distance distribution. A gray dashed vertical line indicates the distance of NGC 6723 quoted in the Harris (1996) compilation $(8.7 \mathrm{kpc})$. We take our estimation of the cluster distance as the mode of the global pdf. Our results are in excellent agreement with literature. Our final estimates are quoted in Fig. 4. To be consistent, we adopt our final estimate of the NGC 6723 distance as that coming from the analysis using reddening computed in situ. In this way we provide a distance of $D=8.7 \mathrm{kpc}$ and a reddening of $E(B-V)=0.083 \mathrm{mag}$ for NGC 6723 . The reddening is slightly larger than the $E(B-V)=0.05$ quoted in Harris (1996).

\section{Abundance ratios}

Abundance ratios were estimated through line-by-line spectrum synthesis calculations for the lines listed in Table A.1. For each line, synthesis calculations were carried with $\mathrm{MOOG}^{5}$, changing the respective elemental abundance until agreement with the observed line was reached. In general, errors in the abundance ratios can be attributed to the adopted $\log (g f)$ and damping constant $\mathrm{C}_{6}$ while constructing the atomic line list. Some tests carried over selected lines, show that changing the $\log (g f)$ value by 0.1 dex produces an abundance variation of the same order for a fixed damping constant.

\footnotetext{
5 Available at http://www . as.utexas.edu/ chris/moog.html
}

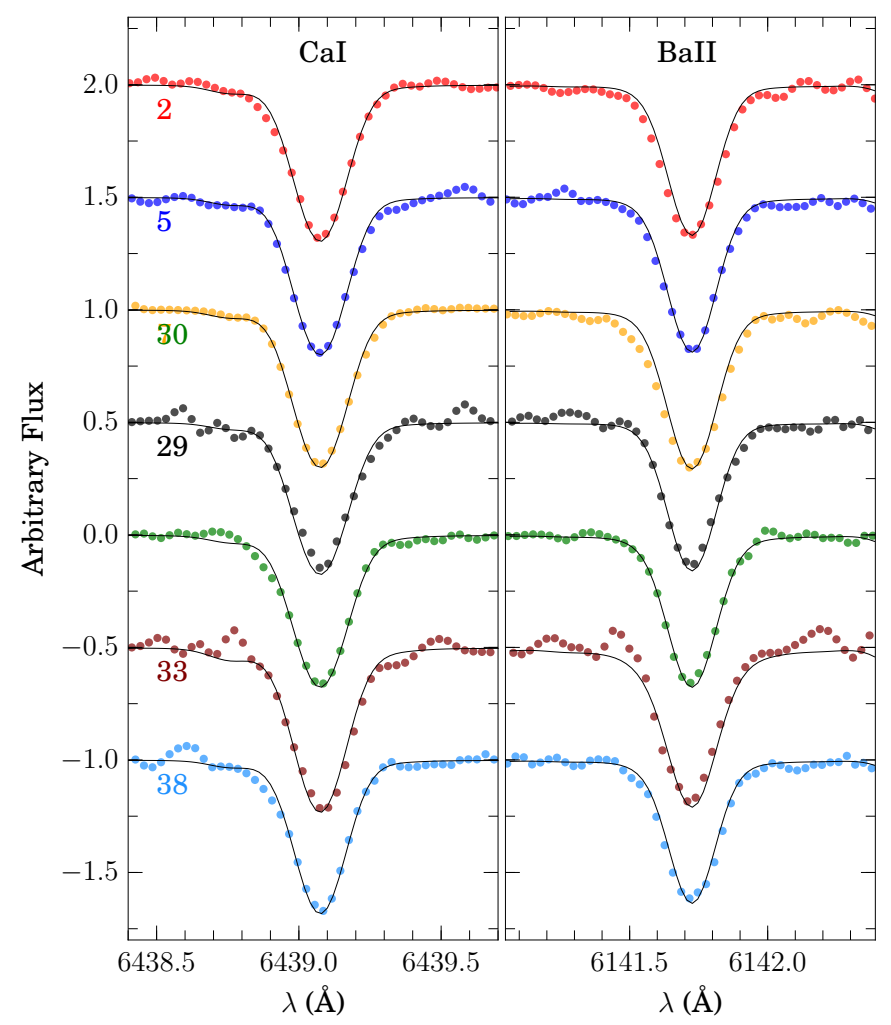

Fig. 5. Ca I $6439.08 \AA$ and Ba II $6141.73 \AA$ lines in the 7 cluster member stars. Observed spectra are depicted by color dots. A solid black line represents the best fit in each case.

In this analysis, molecular lines of $\mathrm{CN}\left(\mathrm{A}^{2} \Pi-\mathrm{X}^{2} \Sigma\right), \mathrm{C}_{2}$ Swan $\left(\mathrm{A}^{3} \Pi-\mathrm{X}^{3} \Pi\right)$, TiO $\left(\mathrm{A}^{3} \Phi-\mathrm{X}^{3} \Delta\right) \gamma$, and $\mathrm{TiO}\left(\mathrm{B}^{3} \Pi-\mathrm{X}^{3} \Delta\right) \gamma^{\prime}$ systems are taken into account. At the typical $T_{\text {eff }}$ of our stars, the main effect of molecule formation in their atmospheres is a lowering of the continuum level. By including molecular features in the spectrum synthesis, we improve the match with observations, alleviating systematics coming from bad continuum definition.

In Fig. 5 we show the fits in the seven sample stars to the Ca I $6439.08 \AA$ and Ba II $6141.73 \AA$ lines. These examples illustrate the general good quality of the fits. Line-by-line abundance ratios are presented for the sample stars in the last seven columns of Table A.1. Final elemental abundances are computed from the average of individual line values. They are listed in Table 5 for each star. The final global cluster abundances are in the last row.

As can be seen from Table $5,[\mathrm{Na} / \mathrm{Fe}]$ abundances show larger spread when compared with the other measured elements. Sodium abundances were determined from two lines at 6154.23 and $6160.75 \AA$ (see Table A.1). During the process of spectrum synthesis for line fitting, we found that these lines are in general small in our sample and not always well defined with respect to the noisy continuum. In fact, the two stars that present better quality are star \#3, which has a combination of high $\mathrm{S} / \mathrm{N}$ ratio and good values of seeing and airmass, and star \#8, which is the noncluster foreground contaminant. This general modest quality for $\mathrm{Na}$ features can explain the larger error bar associated with our final cluster $[\mathrm{Na} / \mathrm{Fe}]$ estimate.

\section{Discussion and conclusions}

We present a high resolution analysis of red giant stars in the bulge globular cluster NGC 6723. Eight targets were originally selected from the $\mathrm{K}$ giant region of the cluster CMD. One of 
Table 5. Final abundance ratios for cluster member stars.

\begin{tabular}{lcccccccc}
\hline \hline Star & {$[\mathrm{O} / \mathrm{Fe}]$} & {$[\mathrm{Na} / \mathrm{Fe}]$} & {$[\mathrm{Mg} / \mathrm{Fe}]$} & {$[\mathrm{Al} / \mathrm{Fe}]$} & {$[\mathrm{Si} / \mathrm{Fe}]$} & {$[\mathrm{Ca} / \mathrm{Fe}]$} & {$[\mathrm{Ti} / \mathrm{Fe}]$} & {$[\mathrm{Ba} / \mathrm{Fe}]$} \\
\hline$\# 1$ & 0.43 & -0.18 & 0.25 & 0.22 & 0.38 & 0.21 & 0.22 & 0.05 \\
$\# 2$ & 0.38 & -0.17 & 0.20 & 0.17 & 0.44 & 0.25 & 0.14 & 0.20 \\
$\# 3$ & 0.15 & 0.39 & 0.25 & 0.50 & 0.39 & 0.23 & 0.21 & 0.26 \\
$\# 4$ & 0.42 & -0.20 & 0.23 & 0.30 & 0.32 & 0.37 & 0.36 & 0.10 \\
$\# 5$ & 0.42 & 0.15 & 0.25 & - & 0.34 & 0.36 & 0.33 & 0.30 \\
$\# 6$ & -0.05 & -0.02 & 0.22 & - & 0.33 & 0.29 & 0.27 & 0.40 \\
$\# 7$ & 0.28 & 0.05 & - & 0.34 & 0.32 & 0.36 & 0.13 & 0.20 \\
Mean & $0.29 \pm 0.18$ & $0.00 \pm 0.21$ & $0.23 \pm 0.10$ & $0.31 \pm 0.21$ & $0.36 \pm 0.05$ & $0.30 \pm 0.07$ & $0.24 \pm 0.09$ & $0.22 \pm 0.12$ \\
\hline
\end{tabular}

Notes. Global values and internal errors (dispersion around the mean) are in the last row.

Table 6. Abundance uncertainties induced by a change of $T_{\text {eff }}=100 \mathrm{~K}$, $\Delta \log (g)=0.2 \mathrm{dex}, \Delta v_{\mathrm{t}}=0.2 \mathrm{~km} \mathrm{~s}^{-1}$, and corresponding total error.

\begin{tabular}{lcccc}
\hline \hline Abundance & $\Delta T$ & $\Delta \log (g)$ & $\Delta v_{\mathrm{t}}$ & $\left(\sum x^{2}\right)^{1 / 2}$ \\
\hline$[\mathrm{O} / \mathrm{Fe}]$ & 0.05 & 0.07 & 0.01 & 0.09 \\
{$[\mathrm{Na} / \mathrm{Fe}]$} & 0.07 & -0.01 & -0.02 & 0.07 \\
{$[\mathrm{Mg} / \mathrm{Fe}]$} & 0.03 & 0.00 & 0.00 & 0.03 \\
{$[\mathrm{Si} / \mathrm{Fe}]$} & -0.04 & 0.00 & -0.10 & 0.11 \\
{$[\mathrm{Ca} / \mathrm{Fe}]$} & 0.05 & -0.03 & -0.07 & 0.09 \\
{$[\mathrm{Ti} / \mathrm{Fe}]$} & 0.13 & 0.01 & -0.04 & 0.14 \\
{$[\mathrm{Ba} / \mathrm{Fe}]$} & 0.06 & 0.07 & -0.12 & 0.15 \\
{$[\mathrm{La} / \mathrm{Fe}]$} & 0.07 & 0.14 & 0.01 & 0.16 \\
\hline
\end{tabular}

these targets turns out to be a foreground field contaminant, based on its discrepant radial velocity, metallicity, and derived distance. Using the clean cluster sample of seven stars, we derived a metallicity of $[\mathrm{Fe} / \mathrm{H}]=-0.98 \pm 0.08 \mathrm{dex}$ and a heliocentric radial velocity of $v_{\text {hel }}=-96.6 \pm 1.3 \mathrm{~km} \mathrm{~s}^{-1}$ for NGC 6723 . Final mean abundance ratios for eight elements are reported in Table 5.

The odd- $Z$ element sodium shows a solar ratio $[\mathrm{Na} / \mathrm{Fe}]=$ $0.00 \pm 0.21$. From their horizontal branch-based study, Gratton et al. (2015) report a somewhat larger value of $0.13 \pm 0.09$ dex.

The $\alpha$-elements oxygen, magnesium, silicon, and calcium are enhanced by $[\mathrm{O} / \mathrm{Fe}]=0.29 \pm 0.18 \mathrm{dex},[\mathrm{Mg} / \mathrm{Fe}]=$ $0.23 \pm 0.10 \mathrm{dex},[\mathrm{Si} / \mathrm{Fe}]=0.36 \pm 0.05 \mathrm{dex}$, and $[\mathrm{Ca} / \mathrm{Fe}]=$ $0.30 \pm 0.07$ dex, respectively. For comparison, Fullton \& Carney (1996) found values of $[\mathrm{Si} / \mathrm{Fe}]=0.68 \pm 0.13$ and $[\mathrm{Ca} / \mathrm{Fe}]=$ $0.33 \pm 0.13$ dex. On the other hand, these elements seem to be overabundant from the estimates of Gratton et al. (2015); $[\mathrm{O} / \mathrm{Fe}]=0.53 \pm 0.09 \mathrm{dex},[\mathrm{Mg} / \mathrm{Fe}]=0.51 \pm 0.06 \mathrm{dex},[\mathrm{Si} / \mathrm{Fe}]=$ $0.60 \pm 0.08 \mathrm{dex}$, and $[\mathrm{Ca} / \mathrm{Fe}]=0.81 \pm 0.13 \mathrm{dex}$. In this work, these authors speculate whether these high enhancements arise as an effect of age (NGC 6723 is very old, 12-13 Gyr, according to several age estimations; Marín-Franch et al. 2009; Dotter et al. 2010; VandenBerg et al. 2013), but they conclude that at least part of this trend can be due to a systematic effect that produces values from their HB sample that are higher by 0.2 dex than those obtained from RGB stars (as already pointed out in Carretta et al. 2010).

The iron-peak element titanium presents a similar enhancement with $[\mathrm{Ti} / \mathrm{Fe}]=0.24 \pm 0.09 \mathrm{dex}$. The same value is reported in Fullton \& Carney (1996). Aluminium is also enhanced by $[\mathrm{Al} / \mathrm{Fe}]=0.31 \pm 0.21 \mathrm{dex}$, while the s-process barium by $[\mathrm{Ba} / \mathrm{Fe}]=0.22 \pm 0.12 \mathrm{dex}$.

Metal-intermediate bulge globular clusters are old objects encoding important information about the environment conditions in which they were formed. A detailed analysis of

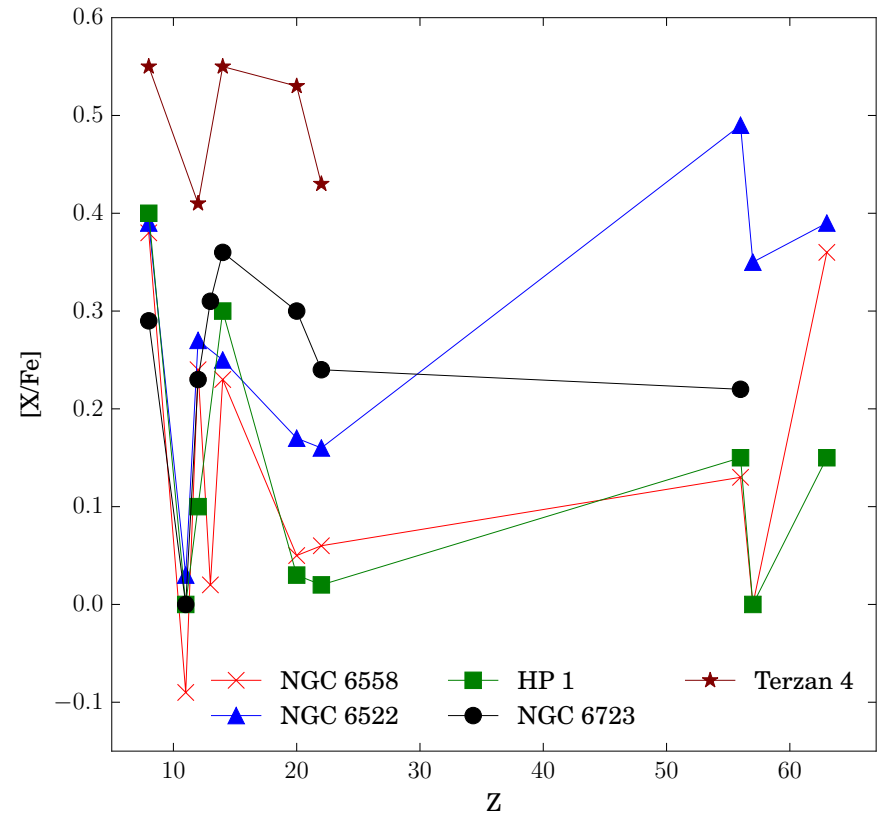

Fig. 6. Chemical enrichment pattern for NGC 6723 (black solid circles). For comparison, other metal-intermediate bulge globular clusters are represented: NGC 6558 (red crosses), NGC 6522 (blue solid triangles), HP1 (green solid squares), and Terzan 4 (black solid stars).

their chemical enrichment patterns can provide valuable information to understand whether or not they share a common origin. Furthermore, studies of other Galactic stellar components (bulge/disk) can benefit from the comparison with clusters as clean independent points in the age-metallicity-distance distributions.

In Fig. 6, the abundance pattern of NGC 6723 is compared with those of other intermediate-metallicity bulge globular clusters. A general similitude is present among NGC $6558([\mathrm{Fe} / \mathrm{H}]=$ -0.97 dex, Barbuy et al. 2007), HP $1([\mathrm{Fe} / \mathrm{H}]=-1.0 \mathrm{dex}$, Barbuy et al. 2006), and NGC $6522([\mathrm{Fe} / \mathrm{H}]=-1.0$ dex, Barbuy et al. 2009). The abundance ratios of NGC 6723 are intermediate between those of the enhanced NGC 6522 and those of NGC 6558 and HP1. Such similarities, suggest a possible common origin for these clusters. In contrast, a more enhanced pattern is displayed by the metal-poor cluster Terzan 4. Given its low metallicity $([\mathrm{Fe} / \mathrm{H}]=-1.60$ dex; Origlia \& Rich 2004), and its somewhat higher velocity components with respect to the average of a sample of bulge globular clusters (Rossi et al. 2015), it is possible that Terzan 4 is a halo cluster currently in the bulge region because it is close to its orbit pericenter. The $\alpha$-enhancement of Terzan 4 is high even compared to the field 

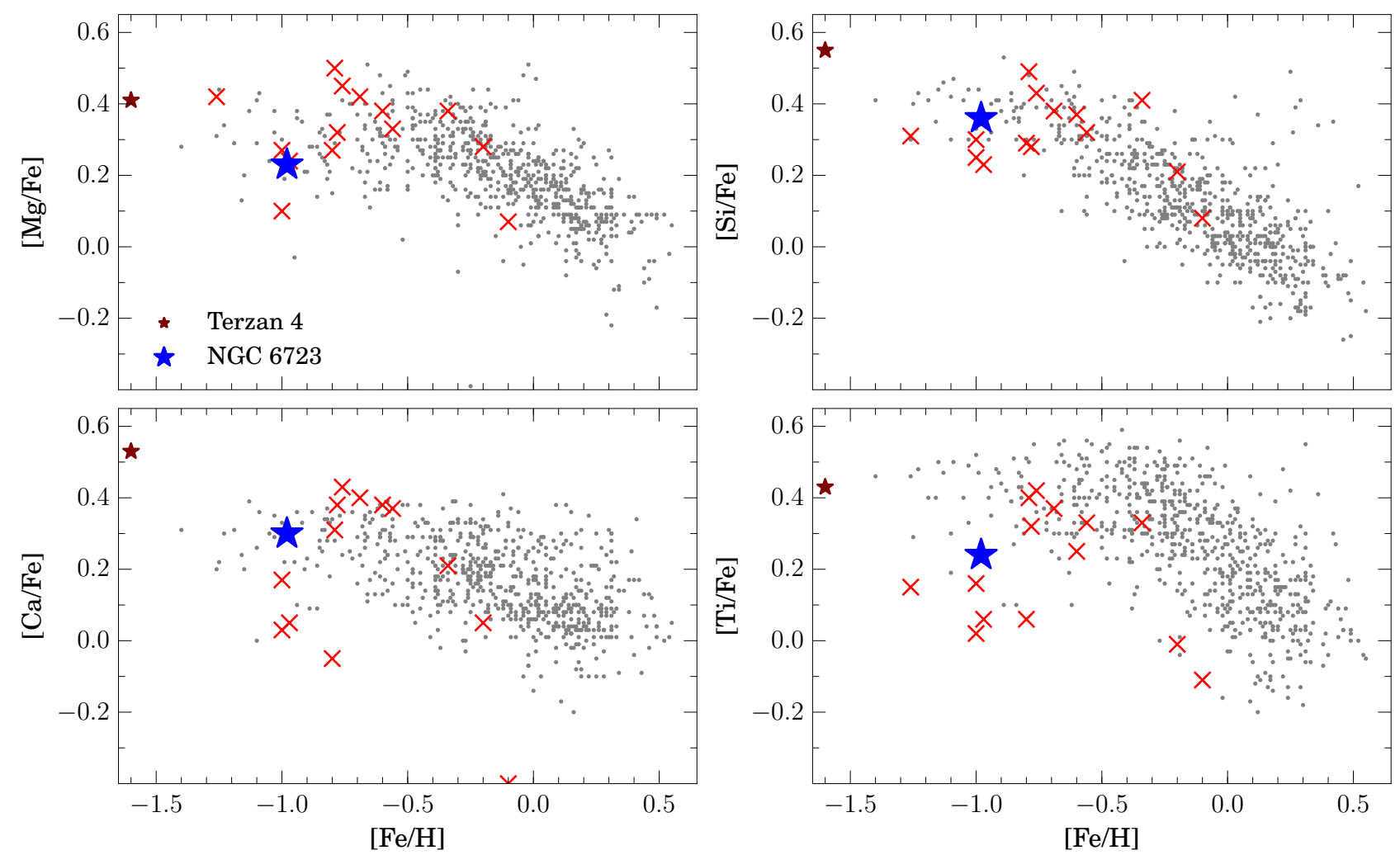

Fig. 7. $[\mathrm{X} / \mathrm{Fe}]$ vs. $[\mathrm{Fe} / \mathrm{H}]$ for NGC 6723 compared with results from literature. Alpha-elements $\mathrm{Mg}, \mathrm{Si}, \mathrm{Ca}$, and iron-peak element Ti are shown in the four panels of the figure. Gray dots depict the $\mathrm{K}$ giant bulge field stars from Gonzalez et al. (2011). A brown star stand for the metalpoor globular cluster Terzan 4 (Origlia \& Rich 2004). Red crosses stand for a number of metal-intermediate and metal-rich clusters from recent literature: NGC 6522 (Barbuy et al. 2009), HP1 (Barbuy et al. 2006), NGC 6558 (Barbuy et al. 2007), NGC 6553 (Alves-Brito et al. 2006), NGC 6528 (Zoccali et al. 2004), Terzan 1 (Valenti et al. 2015), NGC 6441 (Gratton et al. 2007), NGC 6388 (Wallerstein et al. 2007), NGC 6624 and NGC 6569 (Valenti et al. 2011), UKS1 and NGC 6539 (Origlia et al. 2005), NGC 6440 (Origlia et al. 2008), and NGC 6342 (Origlia et al. 2005).

halo stars of Nissen \& Schuster (2010) and Mikolaitis et al. (2014), at the same metallicity.

In order to put our derived abundances in the context of bulge studies, in Fig. 7 we compare these abundances with those of the $\mathrm{K}$ giant bulge sample presented in Gonzalez et al. (2011). As an extra comparison, several metal-rich and metal-intermediate globular clusters are also included from recent literature. The four panels show the $[\mathrm{X} / \mathrm{Fe}]$ vs. $[\mathrm{Fe} / \mathrm{H}]$ distributions for the alpha elements $\mathrm{Mg}, \mathrm{Si}$, and $\mathrm{Ca}$, and $\mathrm{Ti}$. In all cases, a general increase of $[\mathrm{X} / \mathrm{Fe}]$ with decreasing metallicity is observed in the bulge field stars.

The $\alpha$-element ratios of NGC 6723 are in good agreement with those of the bulge field stars. On the other hand, Ti is not following the $\alpha$-elements, and seems to be slightly less enhanced than in the bulge. This discrepancy is still compatible with the larger dispersion of $[\mathrm{Ti} / \mathrm{Fe}]$ values at lower metallicity of the bulge sample.

The other clusters in Fig. 7 compare well with the bulge sample. The agreement in $\mathrm{Mg}$ and $\mathrm{Si}$ is good in the entire metallicity range of the sample. In the case of $\mathrm{Ca}$ and $\mathrm{Ti}$, a fraction of the clusters seem to produce a sequence that is less enhanced and parallel to the bulge sequence. It would be of great interest to further explore this effect to figure out whether it is a spurious effect due to systematic errors, or it is the signature of a different, low alpha population. We emphasize that the comparisons discussed here are only valid at a qualitative level. Indeed, literature determinations for clusters and bulge stars come from a heterogeneous set of studies with different analysis strategies.
In this context, NGC 6723 seems to be characterized by an early prompt chemical enrichment, similar to that attibutted to the the metal-poor portion of the bulge. Finally, the metallicity we found for NGC 6723, of $[\mathrm{Fe} / \mathrm{H}]=-0.98 \mathrm{dex}$, is comparable with the average values $[\mathrm{Fe} / \mathrm{H}]=-1.25 \mathrm{dex}$ (Kunder \& Chaboyer 2008) and $[\mathrm{Fe} / \mathrm{H}]=-1.02$ dex (Pietrukowicz et al. 2012) determined for bulge RR Lyrae stars, as known tracers of old populations.

Further high resolution studies of individual stars in other bulge globular clusters might provide important clues to understand the place that they take in the general assembling of the Milky Way. This might be especially important in the context of the current and future large spectroscopic surveys, exploring Galactic stellar population in increasingly large volumes. Such an observational effort can shade light on important points concerning formation mechanisms of stellar structures and chemodynamical evolution by providing constraints for Galaxy formation model predictions.

Acknowledgements. This work was partially supported by the BASAL CATA through grant PFB-06 CONICYT's PCI program through grant DPI20140066, the Chilean Ministry of Economy through ICM grant to the Millenium Institute of Astrophysics, and the Proyecto Fondecyt Regular 1150345.

\section{References}

Allende Prieto, C., Lambert, D. L., \& Asplund, M. 2001, ApJ, 556, L63 Alonso, A., Arribas, S., \& Martínez-Roger, C. 1999, A\&AS, 140, 261 Alves-Brito, A., Barbuy, B., Zoccali, M., et al. 2006, A\&A, 460, 269 
A. Rojas-Arriagada et al.: High resolution spectroscopic analysis of seven giants in the bulge globular cluster NGC 6723

Baade, D., Meisenheimer, K., Iwert, O., et al. 1999, The Messenger, 95, 15 Babusiaux, C., Gómez, A., Hill, V., et al. 2010, A\&A, 519, A77 Barbuy, B., Zoccali, M., Ortolani, S., et al. 2006, A\&A, 449, 349

Barbuy, B., Zoccali, M., Ortolani, S., et al. 2007, AJ, 134, 1613

Barbuy, B., Zoccali, M., Ortolani, S., et al. 2009, A\&A, 507, 405

Barklem, P. S., Anstee, S. D., \& O’Mara, B. J. 1998, PASA, 15, 336

Barklem, P. S., Piskunov, N., \& O’Mara, B. J. 2000, A\&AS, 142, 467

Bressan, A., Marigo, P., Girardi, L., et al. 2012, MNRAS, 427, 127

Cardelli, J. A., Clayton, G. C., \& Mathis, J. S. 1989, ApJ, 345, 245

Carretta, E., Bragaglia, A., Gratton, R., et al. 2010, ApJ, 712, L21

Coelho, P., Barbuy, B., Meléndez, J., Schiavon, R. P., \& Castilho, B. V. 2005, A\&A, 443, 735

Dinescu, D. I., Girard, T. M., van Altena, W. F., \& López, C. E. 2003, AJ, 125, 1373

Dotter, A., Sarajedini, A., Anderson, J., et al. 2010, ApJ, 708, 698

Fullton, L. K., \& Carney, B. W. 1996, in Formation of the Galactic Halo...Inside

and Out, eds. H. L. Morrison, \& A. Sarajedini, ASP Conf. Ser., 92, 265

Gonzalez, O. A., Rejkuba, M., Zoccali, M., et al. 2011, A\&A, 530, A54

González Hernández, J. I., \& Bonifacio, P. 2009, A\&A, 497, 497

Gratton, R. G., Lucatello, S., Bragaglia, A., et al. 2007, A\&A, 464, 953

Gratton, R. G., Lucatello, S., Sollima, A., et al. 2015, A\&A, 573, A92

Grevesse, N., \& Sauval, A. J. 1998, Space Sci. Rev., 85, 161

Gustafsson, B., Edvardsson, B., Eriksson, K., et al. 2003, in Stellar Atmosphere

Modeling, eds. I. Hubeny, D. Mihalas, \& K. Werner, ASP Conf. Ser., 288, 331

Harris, W. E. 1996, AJ, 112, 1487

Hill, V., Lecureur, A., Gómez, A., et al. 2011, A\&A, 534, A80

Kaufer, A., Stahl, O., Tubbesing, S., et al. 1999, The Messenger, 95, 8

Kraft, R. P., \& Ivans, I. I. 2003, PASP, 115, 143
Kunder, A., \& Chaboyer, B. 2008, AJ, 136, 2441

Lee, J.-W., López-Morales, M., Hong, K., et al. 2014, ApJS, 210, 6

Marín-Franch, A., Aparicio, A., Piotto, G., et al. 2009, ApJ, 694, 1498

Menzies, J. 1974, MNRAS, 168, 177

Mikolaitis, Š., Hill, V., Recio-Blanco, A., et al. 2014, A\&A, 572, A33

Ness, M., Freeman, K., Athanassoula, E., et al. 2013, MNRAS, 430, 836

Nissen, P. E., \& Schuster, W. J. 2010, A\&A, 511, L10

Origlia, L., \& Rich, R. M. 2004, AJ, 127, 3422

Origlia, L., Valenti, E., Rich, R. M., \& Ferraro, F. R. 2005, MNRAS, 363, 897

Origlia, L., Valenti, E., \& Rich, R. M. 2008, MNRAS, 388, 1419

Pietrukowicz, P., Udalski, A., Soszyński, I., et al. 2012, ApJ, 750, 169

Rojas-Arriagada, A., Recio-Blanco, A., Hill, V., et al. 2014, A\&A, 569, A103

Rossi, L. J., Ortolani, S., Barbuy, B., Bica, E., \& Bonfanti, A. 2015, MNRAS, 450, 3270

Rutledge, G. A., Hesser, J. E., \& Stetson, P. B. 1997, PASP, 109, 907

Skrutskie, M. F., Cutri, R. M., Stiening, R., et al. 2006, AJ, 131, 1163

Smith, H. A. 1981, ApJ, 250, 719

Spite, M. 1967, Ann. Astrophys., 30, 211

Stetson, P. B., \& Pancino, E. 2008, PASP, 120, 1332

Valenti, E., Origlia, L., \& Rich, R. M. 2011, MNRAS, 414, 2690

Valenti, E., Origlia, L., Mucciarelli, A., \& Rich, R. M. 2015, A\&A, 574, A80

VandenBerg, D. A., Brogaard, K., Leaman, R., \& Casagrande, L. 2013, ApJ, 775,134

Wallerstein, G., Kovtyukh, V. V., \& Andrievsky, S. M. 2007, AJ, 133, 1373

Zacharias, N., Finch, C., Girard, T., et al. 2010, AJ, 139, 2184

Zoccali, M., Barbuy, B., Hill, V., et al. 2004, A\&A, 423, 507

Zoccali, M., Hill, V., Lecureur, A., et al. 2008, A\&A, 486, 177

Zoccali, M., Gonzalez, O. A., Vasquez, S., et al. 2014, A\&A, 562, A66 


\section{Appendix A: Additional table}

Table A.1. Abundance ratios $[\mathrm{X} / \mathrm{Fe}]$ and line atomic parameters adopted.

\begin{tabular}{|c|c|c|c|c|c|c|c|c|c|c|c|}
\hline \multirow[t]{2}{*}{ Species } & \multirow{2}{*}{$\begin{array}{c}\lambda \\
(\AA)\end{array}$} & \multirow{2}{*}{$\begin{array}{l}\chi_{\mathrm{ex}} \\
(\mathrm{eV})\end{array}$} & \multirow{2}{*}{$\begin{array}{c}\mathrm{C}_{6} \\
\left(\mathrm{~cm}^{6} \mathrm{~s}^{-1}\right)\end{array}$} & \multirow[t]{2}{*}{$\log g f$} & \multicolumn{7}{|c|}{$[\mathrm{X} / \mathrm{Fe}]$} \\
\hline & & & & & $\# 1$ & $\# 2$ & \#3 & $\# 4$ & $\# 5$ & \#6 & $\# 7$ \\
\hline O I & 6300.310 & 0.00 & $3.00 \mathrm{E}-32$ & -9.72 & 0.40 & 0.25 & 0.00 & 0.42 & 0.25 & -0.05 & 0.15 \\
\hline O I & 6363.790 & 4.26 & $3.00 \mathrm{E}-32$ & -2.31 & 0.45 & 0.50 & 0.30 & - & 0.58 & - & 0.40 \\
\hline $\mathrm{NaI}$ & 6154.230 & 0.02 & $5.85 \mathrm{E}-34$ & -10.25 & -0.15 & -0.18 & 0.41 & -0.20 & 0.16 & - & 0.05 \\
\hline $\mathrm{NaI}$ & 6160.753 & 2.10 & $3.55 \mathrm{E}-31$ & -1.59 & -0.20 & -0.15 & 0.37 & - & 0.14 & -0.02 & - \\
\hline $\mathrm{Al} \mathrm{I}$ & 6696.020 & 2.10 & $3.55 \mathrm{E}-31$ & -1.27 & 0.12 & 0.16 & 0.50 & 0.20 & - & - & 0.25 \\
\hline $\mathrm{Al} \mathrm{I}$ & 6698.670 & 3.14 & $6.68 \mathrm{E}-31$ & -1.61 & 0.32 & 0.18 & - & 0.40 & - & - & 0.42 \\
\hline Mg I & 318.720 & 3.14 & $6.67 \mathrm{E}-31$ & -1.95 & 0.25 & 0.20 & 0.25 & 0.23 & 0.25 & 0.22 & - \\
\hline $\operatorname{Mg} I$ & 6765.450 & 5.11 & $3.00 \mathrm{E}-31$ & -1.96 & - & - & - & - & - & - & - \\
\hline $\mathrm{Si}$ I & 142.494 & 5.75 & $3.00 \mathrm{E}-32$ & -1.94 & 0.45 & - & 0.40 & 0.30 & 0.44 & - & 0.35 \\
\hline $\mathrm{Si} \mathrm{I}$ & 45.020 & 5.62 & $00 \mathrm{E}-$ & -1.50 & 0.50 & 0.45 & 0.40 & 0.35 & 0.32 & 0.22 & 0.37 \\
\hline Si I & 55.142 & 5.6 & $00 \mathrm{E}-$ & -1.48 & 0.30 & 0.42 & 0.38 & 0.32 & 0.32 & 0.38 & 0.26 \\
\hline Si I & 37.328 & 5.6 & 1 & -0.93 & - & - & 0.38 & 0.32 & 0.26 & - & - \\
\hline Si I & 43.823 & 5.6 & 1 & -1.19 & 0.36 & - & - & - & - & 0.38 & 0.28 \\
\hline Si I & 21.844 & 5.61 & $21 E-30$ & -1.38 & 0.31 & 0.40 & 0.32 & - & - & - & - \\
\hline $\mathrm{Ca}$ & 56.030 & 5.86 & 39E-30 & -1.20 & 0.31 & - & - & - & - & - & - \\
\hline $\mathrm{CaI}$ & 6161.295 & 2.52 & $5.98 \mathrm{E}-31$ & -2.59 & 0.32 & 0.38 & 0.32 & 0.32 & 0.30 & 0.20 & 0.28 \\
\hline $\mathrm{Ca} \mathrm{I}$ & 6162.167 & 2.52 & $5.00 \mathrm{E}-32$ & -1.29 & 0.25 & 0.25 & 0.27 & 0.38 & 0.30 & 0.35 & 0.32 \\
\hline $\mathrm{CaI}$ & 6166.440 & 1.90 & $2.72 \mathrm{E}-31$ & -0.08 & 0.15 & 0.25 & 0.25 & 0.38 & 0.48 & 0.30 & 0.28 \\
\hline Ca I & 6169.044 & 2.52 & $3.57 \mathrm{E}-31$ & -1.14 & 0.20 & 0.28 & 0.28 & 0.50 & 0.40 & 0.30 & 0.40 \\
\hline $\mathrm{CaI}$ & 6169.564 & 2.52 & $3.57 \mathrm{E}-31$ & -0.77 & 0.20 & 0.30 & 0.22 & 0.42 & 0.40 & 0.45 & 0.44 \\
\hline $\mathrm{CaI}$ & 39.080 & 2.53 & $2.39 \mathrm{E}-31$ & -0.44 & 0.20 & 0.30 & 0.21 & 0.42 & 0.40 & 0.55 & 0.45 \\
\hline $\mathrm{Ca} I$ & 55.605 & 2.53 & $5.12 \mathrm{E}-32$ & 0.30 & 0.20 & 0.20 & 0.23 & 0.33 & 0.33 & 0.25 & 0.30 \\
\hline $\mathrm{Ca} \mathrm{I}$ & 64.679 & 2.5 & $9 \mathrm{E}-32$ & -1.44 & - & - & - & - & - & - & - \\
\hline $\mathrm{Ca} I$ & 71.668 & 2.5 & -31 & -2.42 & 0.20 & 0.35 & 0.23 & 0.30 & 0.38 & 0.25 & 0.40 \\
\hline $\mathrm{Ca} \mathrm{I}$ & & & & -0.71 & 0.15 & 0.10 & 0.15 & 0.30 & 0.30 & 0.10 & 0.27 \\
\hline $\mathrm{CaI}$ & 54 & & & 0.01 & 0.20 & 0.16 & 0.18 & 0.33 & 0.35 & 0.00 & 0.30 \\
\hline $\mathrm{Ca}$ & 08.846 & & & -0.82 & - & - & - & - & - & - & - \\
\hline $\mathrm{CaI}$ & 72.779 & 2.53 & $.05 \mathrm{E}-32$ & -2.50 & 0.15 & 0.16 & 0.18 & - & 0.35 & 0.20 & 0.26 \\
\hline $\mathrm{Ca} \mathrm{I}$ & 717.687 & 0.00 & $2.62 \mathrm{E}-32$ & -4.39 & 0.25 & 0.30 & 0.38 & 0.40 & 0.35 & 0.50 & 0.57 \\
\hline Ti I & 126.224 & 2.71 & $6.19 \mathrm{E}-31$ & -0.58 & 0.22 & 0.14 & 0.23 & 0.30 & 0.39 & 0.30 & 0.09 \\
\hline Ti I & 6258.110 & 1.07 & $2.06 \mathrm{E}-32$ & -1.42 & 0.33 & 0.16 & 0.20 & 0.33 & 0.38 & 0.40 & 0.09 \\
\hline Ti I & 6261.106 & 1.44 & $2.85 \mathrm{E}-32$ & -0.53 & 0.24 & 0.09 & 0.23 & 0.33 & 0.30 & 0.20 & 0.09 \\
\hline Ti I & 303.767 & 1.43 & $4.68 \mathrm{E}-32$ & -0.58 & 0.20 & - & 0.21 & 0.45 & 0.35 & - & - \\
\hline Ti I & 6312.238 & 1.44 & $4.68 \mathrm{E}-32$ & -1.57 & 0.20 & 0.28 & 0.20 & 0.45 & 0.30 & 0.20 & - \\
\hline Ti I & 6336.113 & 1.46 & $4.75 \mathrm{E}-32$ & -1.55 & 0.20 & 0.19 & - & 0.45 & - & - & - \\
\hline Ti I & 6508.154 & 1.44 & $2.79 \mathrm{E}-32$ & -1.74 & - & - & - & - & - & - & - \\
\hline Ti I & & 5.59 & $3.00 \mathrm{E}-32$ & -3.50 & 0.17 & 0.10 & 0.14 & - & 0.30 & 0.27 & 0.25 \\
\hline Ti I & 6556.077 & 1.44 & $2.72 \mathrm{E}-32$ & -1.25 & 0.32 & & 0.32 & - & - & - & - \\
\hline Ti I & 6599.113 & 1.46 & $2.74 \mathrm{E}-32$ & -1.23 & 0.20 & 0.05 & 0.28 & 0.15 & 0.35 & - & 0.20 \\
\hline Ti I & & 0.90 & & -2.08 & 0.15 & 0.10 & 0.10 & 0.29 & 0.30 & 0.27 & 0.06 \\
\hline Ti II & 6491.580 & 0.90 & $2.89 \mathrm{E}-32$ & -1.81 & 0.35 & - & 0.30 & 0.45 & 0.30 & 0.27 & - \\
\hline Ti II & 6559.576 & 3.19 & $3.00 \mathrm{E}-3$ & -3.82 & - & - & - & - & - & - & - \\
\hline Ti II & 6606.970 & 2.06 & $9.86 \mathrm{E}-33$ & -2.18 & - & - & - & - & - & - & - \\
\hline Ba II & 6141.727 & 2.05 & $3.00 \mathrm{E}-32$ & -2.60 & 0.10 & 0.20 & 0.25 & 0.10 & 0.30 & 0.40 & 0.20 \\
\hline Ba II & 6496.908 & 2.06 & $9.63 \mathrm{E}-33$ & -2.99 & 0.00 & 0.20 & 0.26 & 0.10 & 0.30 & 0.40 & 0.20 \\
\hline
\end{tabular}

